\title{
The pulsation-rotation interaction: Greatest hits and the B-side
}

\author{
Rich Townsend \\ Department of Astronomy, University of Wisconsin-Madison, Madison, WI 53706, USA \\ email: townsend@astro.wisc.edu
}

\begin{abstract}
It has long been known that rotation can have an appreciable impact on stellar pulsation - by modifying the usual $\mathrm{p}$ and $\mathrm{g}$ modes found in the non-rotating case, and by introducing new classes of modes. However, it's only relatively recently that advances in numerical simulations and complementary theoretical treatments have enabled us to model these phenomena in any great detail. In this talk I'll review highlights in this area (the 'Greatest Hits'), before considering the flip side (or the 'B-side', for those of us old enough to remember vinyl records) of the pulsation-rotation interaction: how pulsation can itself influence internal rotation profiles.
\end{abstract}

Keywords. stars: oscillations, stars: interiors, stars: rotation, hydrodynamics, waves

\section{Introduction}

Back at the dawn of civilization, the principal medium for distributing music was the vinyl gramophone record. Those who grew up in the vinyl era will recall that a record had two sides - the 'A' side featuring the hit(s) that usually motivated the initial purchase of the record, and the ' $\mathrm{B}$ ' side which contained somewhat more esoteric material often destined to languish in obscurity. $\dagger$

I bring up these facts to draw a strained analogy to the pulsation-rotation interaction in stellar astrophysics. The 'A' side with which we're all familiar comprises the effects of rotation on pulsation; but there's also an accompanying ' $\mathrm{B}$ ' side which considers the influence that pulsation might have on rotation, and indeed the host star's overall evolution. In this contribution I first review the 'greatest hits' on the 'A' side (Sections 2-6), before highlighting some important developments from the ' $\mathrm{B}$ ' side (Section 7).

\section{Perturbative approaches}

Ledoux (1949) and Cowling \& Newcomb (1949) first considered the effects of slow rotation on the oscillation frequencies of a star. As seen from an inertial frame, these can be expressed as

$$
\omega=\omega_{0}+m \Omega\left(1-C_{n, l}\right),
$$

where $\omega_{0}$ is the frequency the mode would have in the absence of rotation, $\Omega$ is the rotation angular frequency (for now, assumed uniform), $C_{n, l}$ the Ledoux constant and $n, l, m$ are the usual mode radial order, harmonic degree and azimuthal order, respectively. The Ledoux constant accounts for the effects of the Coriolis force on the mode. It is usually positive, because the Coriolis force tends to counteract the restoring force on displaced fluid elements for prograde modes $(m>0)$ ), leading to smaller frequencies (vice-versa for

$\dagger$ There are, of course, exceptions: Rock Around the Clock was first released by Bill Haley \& His Comets (a very astronomical band!) on the B-side. 
retrograde modes with $m<0$ ). (Sometimes, however, phenomena such as mode coupling can produce negative $C_{n, l}$; Takata \& Saio, these proceedings, present an example of this). The other term in the parentheses accounts for the Doppler shift in transforming from the co-rotating frame to the inertial frame.

The frequency splitting described by eqn. (2.1) is linear in $m$. This is entirely analogous to the Zeeman splitting of atomic energy levels in a weak magnetic field, and the same first-order perturbation expansion approach underpins the analysis of both phenomena. Moving to more rapid rotation requires a higher-order perturbation expansion. Simon (1969) and a number of subsequent authors extended the formalism to second order in $\Omega$, and Soufi et al. (1998) took it to third order; however, these treatments are significantly more complicated than eqn. (2.1). The value of going to even high orders is moot, because the complexity of the problem becomes unmanageable; equally importantly, there's a point where the effects of rotation can no longer be considered a small perturbation to the non-rotating pulsation equations. Then, so-called 'non-perturbative' approaches are required.

\section{Non-perturbative approaches}

The pulsation equations in a rotating star comprise a 2-dimensional boundary value problem (BVP), with radius $r$ and co-latitude $\theta$ as the independent variables, and the frequency $\omega$ serving as an eigenvalue. Non-perturbative approaches to solving these equations fall into four main groups.

\subsection{Direct methods}

Conceptually, the simplest non-perturbative approach is to approximate the pulsation equations using finite differences on a 2-D $(r, \theta)$ grid. This leads to a large set of algebraic equations, which can be solved using sparse-matrix algorithms. Clement (1998, plus a number of earlier papers) and Savonije et al. (1995) use direct methods, but they have not been more widely adopted for reasons which aren't immediately obvious. (My personal perspective is that the numerical aspects of direct methods are rather daunting).

\subsection{Spectral methods}

Spectral methods expand the angular dependence of solutions as (typically large, but finite) sums of spherical harmonics $Y_{l}^{m}(\theta, \phi)$, with the same azimuthal orders but harmonic degrees $l=|m|,|m|+2,|m|+4, \ldots$ for even-parity modes, and $l=|m|+1,|m|+$ $3,|m|+5, \ldots$ for odd-parity modes. This reduces the pulsation equations to a 1-D BVP, with the expansion coefficients being the unknowns. This BVP is solved using the same general techniques as in the non-rotating case (see, e.g., Townsend \& Teitler 2013, and references therein), although the computational cost is much higher.

Spectral methods are the oldest of the approaches described here, dating back to the pioneering work on the oscillations of rotating polytropes by Durney \& Skumanich (1968). They have become increasingly popular in recent years (e.g., Lee \& Baraffe 1995; Reese et al. 2006; Ouazzani et al. 2012), perhaps driven by the advent of inexpensive highperformance computing hardware. One criticism leveled at spectral methods is that the truncation of the spherical-harmonic expansion necessarily makes them approximate. This is technically true, but only inasmuch as any numerical solution of a system of differential equations is an approximation. The number of spherical harmonics can always be made sufficiently large to achieve the desired level of accuracy - much as the grid spacing in a finite-difference method can always be made sufficiently small. 


\subsection{Ray-tracing methods}

Ray-tracing methods treat the pulsation equations in an asymptotic limit analogous to the geometric limit of optics. The resulting eikonal equation is integrated using the method of characteristics (at a computational cost much smaller than the direct or spectral methods described above), to find the ray paths followed by short-wavelength acoustic waves through a rotating model star. The properties of these rays can be studied using Poincaré surface sections, which mark each passage of a ray through a fixed-radius surface with a point plotted in the $\theta-k_{\theta}$ (polar wavenumber) plane.

Lignières \& Georgeot (2008) use ray tracing to show that the acoustic properties of rapidly rotating polytropes fall into three main groups: rays bouncing internally between mid-latitude surface regions in the northern and southern hemispheres, rays confined to a surface layer at all latitudes, and rays completely filling the interior. These groups correspond, respectively, to the three classes of global p mode explored by Reese et al. (2009) using a spectral method: island modes (small $l-|m|$ ), whispering gallery modes (large $l-|m|$ ), and chaotic modes (intermediate $l-|m|)$.

\subsection{Traditional approximation}

The traditional approximation neglects the horizontal component of the angular velocity vector when evaluating the Coriolis force. Originating in the geophysical literature (see Eckart 1960), it is a reasonable approximation in radiative regions when both the oscillation frequency and the rotation frequency are much smaller than the local Brunt-Väisälä frequency $N$ - that is, for intermediate- and high-order g-modes.

If the centrifugal force and the gravitational potential perturbations are also neglected, the traditional approximation brings a huge simplification to the pulsation equations: it allows them to be separated in $r$ and $\theta$. Solution then proceeds as in the non-rotating case, with only two substantive changes: spherical harmonics $Y_{l}^{m}(\theta, \phi)$ are replaced by so-called Hough functions $\Theta(\theta) \exp (\mathrm{i} m \phi)$ (Hough 1897; see also Townsend 2003a), which are the eigenfunctions of Laplace's tidal equation, and $l(l+1)$ terms are replaced by the corresponding eigenvalues $\lambda$ of the tidal equation.

These eigenvalues depend on the 'spin parameter' $\nu=2 \Omega / \omega_{\mathrm{c}}$, where

$$
\omega_{\mathrm{c}} \equiv \omega-m \Omega
$$

is the oscillation frequency in the co-rotating frame. In the limit $\nu \rightarrow 0, \lambda \rightarrow l(l+1)$; but for $\nu \gtrsim 1, \lambda$ departs markedly from its non-rotating value. It is this 'inertial regime' which remains inaccessible to perturbative approaches. (As an aside: the significance of $\nu$ is that it measures by how much the star turns during one oscillation cycle - and thus to what extent a given mode is 'aware' its frame of reference is rotating).

There's an important caveat to the traditional approximation: it neglects the possibility of resonant coupling between pairs of modes with the same $m$. Lee \& Saio (1989) demonstrated that these resonances are manifested in avoided crossings between the mode frequencies; but in the traditional approximation the avoided crossings are transformed into ordinary crossings, because the resonances are suppressed. This explains why the traditional approximation is unable to reproduce the properties of the rosette modes found in rapidly rotating polytropes by Ballot et al. (2012); as Takata \& Saio (these proceedings) demonstrate, these modes result from near-degeneracies in the non-rotating frequency spectrum, which are then pushed into resonance by the Coriolis force. 


\section{The rapidly rotating limit}

The complexity of the higher-order perturbative approaches (Section 2) might make us concerned that understanding oscillations in rapidly rotating stars is going to be extremely difficult. Fortunately, however, this often turns out not to be the case; new regularities appear in the frequency spectra, in just the same way that atomic energy levels become regular again in the strong-field limit (the Paschen-Back effect).

\subsection{Regularities in p-mode spectra}

Based on fitting to spectral-method calculations, but also guided by insights from ray tracing, Reese et al. (2009) propose an empirical formula for the regularities seen in the frequency spectra of island acoustic modes:

$$
\omega_{\mathrm{c}} \approx \tilde{n} \tilde{\Delta}_{\tilde{n}}+\tilde{l} \tilde{\Delta}_{\tilde{l}}+m^{2} \tilde{\Delta}_{\tilde{m}}+\tilde{\alpha} .
$$

Here, the various $\tilde{\Delta}$ terms together with $\tilde{\alpha}$ are obtained from least-squares fitting to the calculated frequency spectra, while $\tilde{n}$ and $\tilde{l}$ are new mode indices which correspond to the number of eigenfunction nodes along and parallel to the ray paths stretching between the two mid-latitude surface endpoints of island modes (see Fig. 3 of Reese et al. 2009). These indices can be related to the radial order and harmonic degree of the modes' non-rotating counterparts via

$$
\tilde{n}=2 n, \quad \tilde{l}=\frac{l-|m|}{2}
$$

for even-parity modes, and by

$$
\tilde{n}=2 n+1, \quad \tilde{l}=\frac{l-|m|-1}{2}
$$

for odd-parity modes.

A hand-waving narrative can be used explain the form of eqn. (4.1). The terms proportional to $\tilde{n}$ and $\tilde{l}$ appear by direct analogy to the standard asymptotic expression for p-mode frequencies in a non-rotating star (e.g., Aerts et al. 2010, their eqn. 3.216), which contains terms linear in $n$ and $l$. The term proportional to $m^{2}$ accounts for the bulk effects of the centrifugal force, which do not depend on the sign of $m$. Finally, the $\tilde{\alpha}$ term accounts for the phase of waves at the stellar surface.

\subsection{Regularities in g-mode spectra}

Ballot et al. (2010) explore g modes in rotating stars using a spectral method. Although these authors' focus is primarily on the inadequacies of perturbative approaches in the inertial regime $(\nu>1)$, their Figs. 4 and 5 illustrate quite strikingly that, as with the $\mathrm{p}$ modes above, new regularities appear in the g-mode frequency spectrum at rapid rotation rates.

These are a consequence of mode trapping in an equatorial waveguide. When $\nu>1$ the Coriolis force prevents g modes from propagating outside of the region $|\cos \theta| \leqslant \nu^{-1}$. In the limit $\nu \gg 1$, the trapping can be modeled using an asymptotic treatment of Laplace's tidal equation first developed by Matsuno (1966). The eigenvalue $\lambda$ (cf. Section 3.4) is found as

$$
\lambda \approx \begin{cases}\nu^{2}\left(2 l_{\mu}-1\right)^{2} & l_{\mu} \geqslant 1, \\ m^{2} & l_{\mu}=0\end{cases}
$$

(e.g., Bildsten et al. 1996), where the mode index $l_{\mu}$ counts the number of zonal nodes in the radial displacement eigenfunction. This index is related to the harmonic degree and 
azimuthal order of the modes' non-rotating counterparts via

$$
l_{\mu}=l-|m|
$$

for prograde and axisymmetric $(m=0)$ modes, and

$$
l_{\mu}=l-|m|+2
$$

for retrograde modes.

The co-rotating frequencies of high-order g modes depend on $\lambda$ via

$$
\omega_{\mathrm{c}} \approx \frac{\sqrt{\lambda}}{\pi(n+\alpha)} \int \frac{N}{r} \mathrm{~d} r .
$$

This is just the usual asymptotic expression (e.g., eqn. 3.235 of Aerts et al. 2010) with $\sqrt{l(l+1)}$ replaced by $\sqrt{\lambda}$. Combining this with eqn. (4.2) and solving for $\omega_{\mathrm{c}}$ yields

$$
\omega_{\mathrm{c}} \approx\left[\frac{2 \Omega\left(2 l_{\mu}-1\right)}{\pi(n+\alpha)} \int \frac{N}{r} \mathrm{~d} r\right]^{1 / 2}
$$

for modes with $l_{\mu} \geqslant 1$, and

$$
\omega_{\mathrm{c}} \approx \frac{m}{\pi(n+\alpha)} \int \frac{N}{r} \mathrm{~d} r
$$

for the $l_{\mu}=0$ modes, which are sometimes labeled equatorial Kelvin modes (Townsend 2003a). These Kelvin modes have an azimuthal phase velocity $\omega_{\mathrm{c}} / m$ that doesn't depend on $m$; they may therefore be able to explain the unusual uniformly spaced low-frequency modes seen in some $\delta$ Scuti stars (e.g., KIC 8054146; see Breger, these proceedings).

These expressions indicate that a mode multiplet with a given $n, l$ and $-l \leqslant m \leqslant l$ will reorganize itself into $l+2$ distinct frequencies, corresponding to the permitted indices $0 \leqslant l_{\mu} \leqslant l+1$. One corresponds to the Kelvin mode $m=l$. Of the remaining $l+1$ distinct frequencies, the lowest corresponds to the $m=l-1$ mode, the next $l-1$ are made from pairings between prograde and retrograde modes with the same $l_{\mu}$, and the final, highest frequency corresponds to the $m=-1$ mode. This is exactly the pattern seen in Figs. 4 and 5 of Ballot et al. (2010) in the rapidly rotating limit (note that these authors' sign convention for $m$ is reversed), confirming the analysis here.

\subsection{Inertial modes}

The foregoing discussion focuses on pulsation modes of rapidly rotating stars that have oscillatory counterparts in the non-rotating limit (in other words, $\omega_{c}^{2}$ remains greater than zero and deforms continuously as $\Omega$ is varied between the two limits). However, the Coriolis force introduces additional classes of 'inertial' mode whose non-rotating counterparts have $\omega^{2} \leqslant 0$. Regions with negative $N^{2}$ can be stabilized against perturbations with wavenumber $\mathbf{k}$ if

$$
N^{2} k_{\perp}^{2}>-(2 \Omega \cdot \mathbf{k})^{2}
$$

(here, $k_{\perp}$ is the horizontal component of $\mathbf{k}$ ). Convective fluid motions are then transformed into oscillatory motions, with the Coriolis force serving as the restoring force.

The Coriolis force likewise transforms the trivial toroidal modes having $\omega^{2}=0$ in the non-rotating limit into $\mathrm{r}$ (Rossby) modes with $\omega_{c}^{2}>0$. The restoring force for $\mathrm{r}$ modes does not depend on the stellar structure, but instead comes from conservation of total vorticity (see Saio 1982 for an illuminating discussion). In the slowly rotating limit their frequencies are given by

$$
\omega_{c}=-\frac{2 m \Omega}{l(l+1)} .
$$


The opposite signs of $\omega_{\mathrm{c}}$ and $m$ in this expression tell us that $\mathrm{r}$ modes are always retrograde in the co-rotating frame. Toward more rapid rotation the frequencies depart from this formula, acquiring a dependence on the underlying stellar structure. The departures are especially pronounced for the $m=-l$ modes, which behave more like $\mathrm{g}$ modes and indeed follow the equatorial waveguide expression (4.3) with $l_{\mu}=1$. These 'mixed gravity-Rossby' modes pair up with the $m=l-1$ modes of frequency multiplets, so the number of distinct frequencies in the multiplets remains unchanged. Due to their g-mode character they can be excited by the $\kappa$ mechanism, as demonstrated for instance in Townsend (2005).

\section{Mode visibilities}

Rapid rotation tends to reduce the photometric visibility of oscillations. For p modes with small $l-|m|$ this is a consequence of their transformation into island modes, whose surface amplitudes are appreciable only at mid-latitudes; for g modes, this results from confinement in the equatorial waveguide. As a result, it becomes challenging to detect modes photometrically in rapidly rotating stars - and even if modes can be seen, they are subject to strong selection effects (which at low frequencies strongly favor equatorial Kelvin modes; see Townsend 2003b). For an overview of recent developments in this area, see Daszyńska-Daszkiewicz et al. (2007) and Reese et al. (2013).

\section{Differential rotation}

So far I've focused on the simple case of uniform rotation. However, there's evidence from a multitude of sources that the internal rotation of stars is differential in $r$ and/or $\theta$ (the most well-known example is the Sun; see Thompson et al. 2003). Both perturbative and non-perturbative approaches can readily be adapted to handle differential rotation; but here, let's focus on an even simpler analysis.

If the Coriolis and centrifugal forces are neglected, then the only effect of rotation is the Doppler shift in transforming between co-rotating and inertial frames. However, in a differentially rotating star the notion of a global co-rotating frame must be replaced by a continuous sequence of local frames which rotate with angular frequency $\Omega(r, \theta)$. Via equation (3.1), the co-rotating frequency is thus a function of position in the star, and in principle can vanish wherever $m \Omega=\omega$. At these locations, known as 'critical layers' (see Mathis et al., these proceedings), the dispersion relation for low-frequency gravity waves

$$
k_{r}^{2} \approx k_{\perp}^{2} \frac{N^{2}}{\omega_{\mathrm{c}}^{2}}
$$

suggests that the radial component $k_{r}$ of the wavenumber should diverge. In reality, what will happen is that the wavelength near a critical layer becomes so short that significant radiative dissipation occurs. Thus, critical layers in a differentially rotating star can play a pivotal role in governing mode excitation and damping.

\section{Wave transport of angular momentum}

Let's now flip the record to the 'B' side, and discuss what impact stellar pulsations might have on their host star. Just as waves transport energy, they also transport momentum. A series of papers by Ando $(1981,1982,1983)$ first considered how the angular momentum transported by non-axisymmetric waves alters the internal rotation profile of 
a star. To model this process we can perform a Reynolds decomposition of the azimuthal momentum equation to find the angle-averaged volumetric torque as

$$
\frac{\partial}{\partial t}\left\langle\varpi \overline{\rho v}_{\phi}\right\rangle=-\frac{1}{4 \pi r^{2}} \frac{\partial}{\partial r} L_{J}-\frac{\partial}{\partial t}\left\langle\varpi \overline{\rho^{\prime} v_{\phi}^{\prime}}\right\rangle-\left\langle\overline{\rho^{\prime} \frac{\partial \Phi^{\prime}}{\partial \phi}}\right\rangle .
$$

Here $\varpi \equiv \sin \theta$, while the overline denotes the average over azimuth and the angled brackets the average over co-latitude. The bracketed term on the left-hand side represents the angular momentum in a spherical shell of unit thickness. The first term on the right-hand side is the torque arising from the gradient of the wave angular momentum luminosity $L_{J}$; the second term is the rate-of-change of the angular momentum stored in wave motions; and the third term is the gravitational torque.

Focusing on the first term, the angular momentum luminosity (that is, the net amount of angular momentum flowing through a spherical surface in unit time) is given by

$$
L_{J}=4 \pi r^{2}\left\langle\varpi\left(\bar{\rho} \overline{v_{r}^{\prime} v_{\phi}^{\prime}}+\bar{v}_{\phi} \overline{\rho^{\prime} v_{r}^{\prime}}\right)\right\rangle,
$$

to second order in the pulsation amplitude (e.g., Lee \& Saio 1993). The first term in the parentheses is the Reynolds stress generated by the radial and azimuthal fluid motions. It vanishes in the case of pure standing waves, because $v_{r}^{\prime}$ and $v_{\phi}^{\prime}$ are exactly 90 degrees out of phase. However, departures from this strict phase relation arise when waves acquire a propagative component - either due to non-adiabatic effects, or from wave leakage at the outer boundary. In the non-adiabatic case, the Reynolds stress term for prograde modes transports angular momentum from excitation regions to dissipation regions (vice-versa for retrograde modes; see Ando 1986).

The second term in the parentheses of eqn. (7.1) is the eddy mass flux. Again, this term vanishes for pure standing waves, but becomes non-zero when waves acquire a propagative component. Shibahashi (these proceedings) proposes the intriguing hypothesis that the eddy mass flux of g modes, leaking through the surface layers of Be stars, can transport the angular momentum necessary to build a quasi-Keplerian disk. The transport is particularly effective in the outer layers because the Eulerian pressure perturbation $\rho^{\prime}$ is large due to the steep density gradient there.

Stellar evolution calculations which include wave transport of angular momentum have so far focused primarily on stochastically excited modes (see, e.g., Talon 2008 and references therein). However, simple estimates of transport by overstable global modes suggest that they can have a significant impact on rotation profiles over timescales which are evolutionarily short (Townsend 2009). Interest in this topic is steadily growing; just this year a number of new papers have appeared exploring topics such as wave transport in massive stars (Rogers et al. 2013) and pre-main sequence stars (Charbonnel et al. 2013), and the interaction between wave transport and critical layers (Alvan et al. 2013).

\section{Summary}

To summarize this review, I'd like to highlight an encouraging trend. Much of the recent progress in understanding the pulsation-rotation interaction has been driven by numerical simulations. However there have been multiple parallel efforts to develop complementary theoretical narratives for the interaction. These have allowed us to retain a firm grasp on what's really going on in the simulations, and also reassured us that the rapidly rotating limit might not be as difficult to understand as we once thought. Let's ensure this trend does not disappear in the future, by always remembering the wonderful adage by Hamming (1987): 'the purpose of computing is insight, not numbers'. 


\section{Acknowledgements}

I acknowledge support from NSF awards AST-0908688 and AST-0904607 and NASA award NNX12AC72G. I'd also like to thank the IAU, and the SOC/LOC, for helping support my travel to this meeting.

\section{References}

Aerts, C., Christensen-Dalsgaard, J., \& Kurtz, D. W. 2010, Asteroseismology (Dordrecht: Springer)

Alvan, L., Mathis, S., \& Decressin, T. 2013, A\&A, 553, A86

Ando, H. 1981, MNRAS, 197, 1139

Ando, H. 1982, A\&A, 108, 7

Ando, H. 1983, PASJ, 35, 343

Ando, H. 1986, A\&A, 163, 97

Ballot, J., Lignières, F., Reese, D. R., \& Rieutord, M. 2010, A\&A, 518, A30

Ballot, J., Lignières, F., Prat, V., Reese, D. R., \& Rieutord, M. 2012, ASP-CS, 462, 389

Bildsten, L., Ushomirsky, G., \& Cutler, C. 1996, ApJ, 460, 827

Charbonnel, C., Decressin, T., Amard, L., Palacios, A., \& Talon, S. 2013, A\&6A, 554, A450

Cowling, T. G. \& Newing, R. A. 1949, ApJ, 109, 149

Daszyńska-Daszkiewicz, J., Dziembowski, W. A., \& Pamyatnykh, A. A. 2007, AcA, 57, 11

Durney, B. \& Skumanich, A. 2008, ApJ, 152, 255

Eckart, C. 1960, Hydrodynamics of Oceans and Atmospheres (Oxford: Pergamon Press)

Hamming, R. W. 1987, Numerical Methods for Scientists and Engineers (Dover, New York)

Hough, S. S. 1898, Phil. Trans. Roy. Soc. Lon., 191, 139

Ledoux, P. 1949, Mem. Soc. R. Sci. Liège, 4(9), 3

Lee, U. \& Baraffe, I. 1995, A\&A, 301, 419

Lee, U. \& Saio, H. 1993, MNRAS, 261, 415

Lignières, F. \& Georgeot, B. 2008, Phys. Rev. E, 78, 016215

Matsuno, T. 1966, J. Meteorol. Soc. Japan, 44, 25

Ouazzani, R.-M., Dupret, M.-A., \& Reese, D. R. 2012, A\&AA, 547, A75

Reese, D. R., Lignières, F., \& Rieutord, M. 2006, A\&A, 455, 621

Reese, D. R., MacGregor, K. B., Jackson, S., Skumanich, A., \& Metcalfe, T. S. 2009, A\&A A, 506, 189

Reese, D. R., Prat, V., Barban, C., van 't Veer-Menneret, C., \& MacGregor, K. B. 2013, A\&A, 550, A77

Rogers, T. M., Lin, D. N. C., McElwaine, J. N., \& Lau, H. H. B. 2013, ApJ, 772, 21

Saio H. 1982, ApJ, 256, 717

Savonije, G. J., Papaloizou, J. C. B., \& Alberts, F. 1995, MNRAS, 277, 471

Simon, R. 1969, A\&A, 2, 390

Soufi, F., Goupil, M.-J., \& Dziembowski, W. A. 1998, A\&A, 334, 911

Talon, S. 2008, in: C. Charbonnel \& J.-P. Zahn (eds.), Stellar Nucleosynthesis: 50 years after B2FH, EAS Publ. Ser., 32, 81

Thompson, M. J., Christensen-Dalsgaard, J., Miesch, M. S., \& Toomre, J. 2003, ARAA, 41, 599

Townsend, R. H. D. 2003a, MNRAS, 340, 1020

Townsend, R. H. D. 2003b, MNRAS, 344, 125

Townsend, R. H. D. 2005, MNRAS, 364, 573

Townsend, R. H. D. 2009, AIP-CS, 1170, 355

Townsend, R. H. D. \& Teitler, S. A. 2013, MNRAS, 435, 3406 